\title{
mm-VLBI observations of SiO masers: Further support for radiative pumping and rotation of the inner shell.
}

\author{
Jean-François Desmurs, Valentín Bujarrabal, Francisco Colomer, and \\ Javier Alcolea
}

Observatorio Astronómico Nacional (IGN), Spain

\begin{abstract}
We have performed VLBA observations of the $\mathrm{SiO} v=1$ and $v=2 J=1-0$ masers in two AGB stars, TX Cam and IRC +10011. We confirm the ring-like spatial distribution, previously found in several AGB objects, as well as the tangential polarization pattern, already reported for TX Cam. Both properties, that seem to be systematic in this kind of objects, are characteristic of radiatively pumped $\mathrm{SiO}$ masers. On the contrary, we do not confirm the previous report on the spatial coincidence between the $J=1-0 v=1$ and 2 masers, a result that would have argued in favor of collisional pumping. We find that both lines sometimes arise from nearby spots, typically separated by 1-2 mas, but are rarely coincident. The discrepancy with previous results is explained by the very high spatial resolution of our observations, $\sim 0.5$ mas, an order of magnitude better than in the relevant previously published experiment. Moreover, we have been able to measure a probable rotation of the inner shell of a few $\mathrm{km} / \mathrm{s}$. Rotation of circumstellar shells is assumed by the most convincing models explaining the drastic change of symmetry between the AGB envelopes (spherical symmetry) and Proto Planetary Nebulae (axial symmetry).
\end{abstract}

\section{Introduction}

$\mathrm{SiO}$ maser emission at $7 \mathrm{~mm}$ wavelength $(v=1$ and $v=2, J=1-0$ transitions around $43 \mathrm{Ghz}$ ) has been observed in AGB stars with very high spatial resolution by means of VLBI techniques, yielding important results in relation with the not yet well understood pumping mechanism. The $7 \mathrm{~mm}$ maser emission regions are found to be distributed in a number of spots forming a ring-like structure at about 2-3 stellar radii (see Diamond et al. 1994, Miyoshi et al., 1994 and Greenhill et al., 1995).

As we will see the study of the inner shell by means of $\mathrm{SiO}$ maser lines is in particular useful to understand the evolution from the spherical symmetry of AGB envelopes toward the conspicious axial shape of Planetary Nebulae (PNe), that seems to be already present as soon as the stars leave the AGB (Bujarrabal et al. 1992, Trammell et al. 1994).

\section{Arguments in favor of Radiative Pumping}

Our high-quality VLBA maps confirm that both $v=1$ and $v=2 \quad J=1-0$ maser transitions arise from ring-like strutures, at a distance from the center of $\sim$ 
14 mas $\left(8 x 10^{13} \mathrm{~cm}\right.$ assuming a distance of $\left.\sim 350 \mathrm{pc}\right)$ for TX Cam, and $\sim 11$ mas $\left(\sim 10^{14} \mathrm{~cm}\right.$ assuming a distance of $\left.\sim 500 \mathrm{pc}\right)$ for IRC+10011. Our maps show that $v=1$ and $v=2$ spots are systematically not coincident. In TX Cam we identify, from the integrated flux maps (see Figure 3 in Desmurs et al., 2000 ), about 17 features in both $v=1$ and $v=2$ transitions, out of which only one appears to be spatially coincident, within the resolution of our observation. In the case of IRC+10011, we find about 13 features, 4 of which could be coincident (see Figure 2, in Desmurs et al., 2000). In most cases in which spots of these lines are nearby, there is a systematical shift between them, of about 1-2 mas, being the $v=2$ ones placed in a layer slightly closer to the star. The spatial coincidence observed previously by Miyoshi et al. (1994) is then not confirmed. This disagreement can be explained by the fact that the shift that we measured is significally smaller ( 1 or 2 mas) than the resolution beam of the observations in Miyoshi et al (7 mas).

The bulk of the existing results on $\mathrm{SiO}$ maser emission from evolved stars strongly support radiative pumping. The spatial shell-like distribution of the flux in a thin ring, the tangential polarization pattern of $v=1$ and $2 J=1-0 \mathrm{SiO}$ masers, and the shift in their spatial distributions are intrinsically related with the radiative pumping schemes. Indeed, radiative models predicted, well before the observations were made, (Bujarrabal et al., 1981) that such properties should be systematically observed.
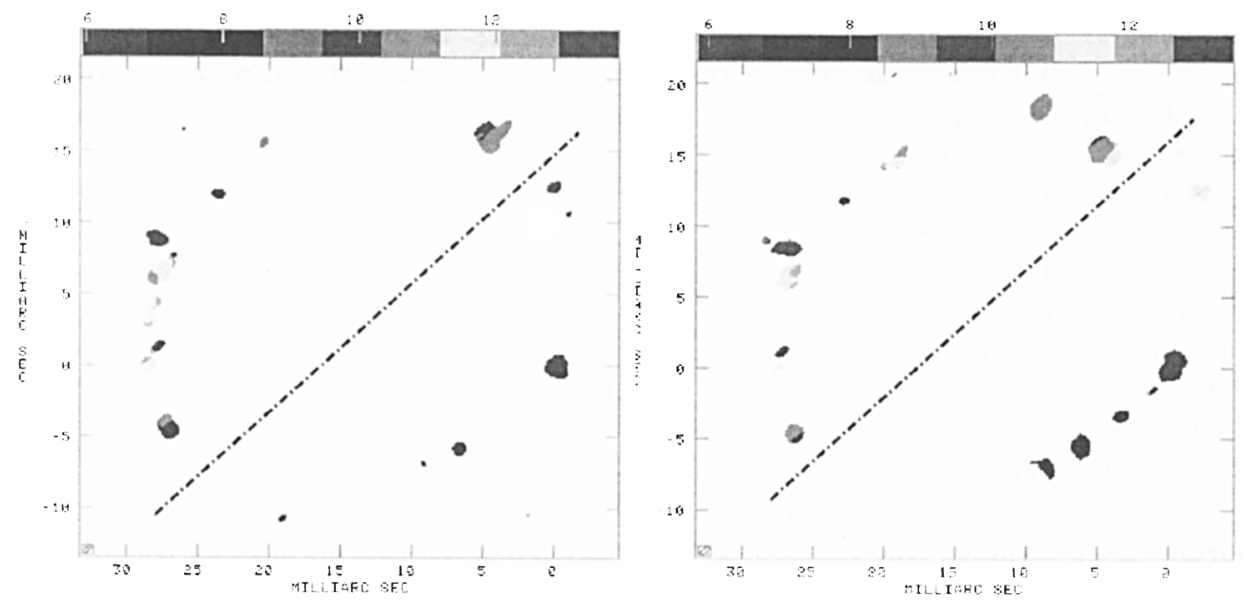

Figure 1. Distribution of velocities in TX Cam, for $v=1$ (Left) and $v=2$ (Right), $J=1-0$ (systemic velocity $\sim 9 \mathrm{~km} / \mathrm{s}$ ).

\section{Rotation of the inner circumstellar shell}

The most convincing present models of post-AGB evolution (see e.g. Soker $1998 \mathrm{a}, \mathrm{b})$ assume a significant rotation in the inner AGB envelope. When the star ejects its extended atmosphere (and the post-AGB phase starts), its radius 

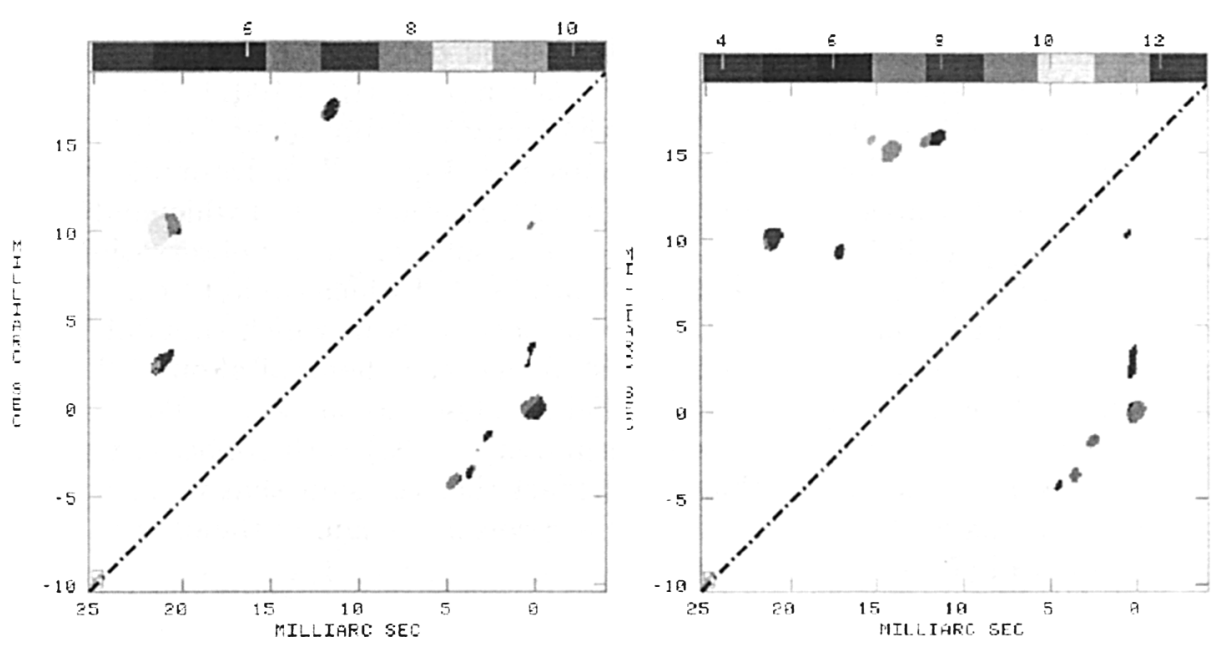

Figure 2. Distribution of velocities in IRC+10011, for $v=1$ (Left) and $v=2$ (Right), $J=1-0$ (systemic velocity $\sim 8 \mathrm{~km} / \mathrm{s}$ )

strongly decreases and a reaccretion of a part of the circumstellar envelope can take place. Fast bipolar jets then appear by interaction of the infalling material with the stellar magnetic field (a process similar to that explaining the outflows in forming stars), provided that the inner circumstellar shells have a significant excess of angular momentum. The subsequent shock interaction between the jets and the envelope ejected during the previous AGB phase would lead to the observed axial shapes of PNe. This theory explains the observed axial symmetry of PPNe and the very collimated post-AGB jets. It also explains the peculiar abundances found in the atmospheres of certain post-AGB stars, that would be affected by the abundances of the reaccreted material (e.g. Van Winckel et al. 1995), as well as the very high energy and momentum of the bipolar outflows in PPNe, that cannot be explained by radiation pressure and seem to require a release of gravitational energy (Bujarrabal et al. 1998).

This process is only possible if a significant rotation takes place in the inner circumstellar shells, during the AGB phase, which should be due to dynamical interaction of the stellar atmosphere or inner envelope with a stellar companions or planets (see detailed models by Siess \& Livio 1999).

From our observations of the $v=1$ and $v=2 \quad J=1-0$ maser line in TX Cam and IRC+10011, we have been able to measure a rotation of the inner shell emiting the $\mathrm{SiO}$ masers in both sources and for both lines. For TX Cam, see Figure 1, the systemic velocity of the source is $9 \mathrm{~km} / \mathrm{s}$. We can see that the emission emited in the eastern half of the ring appears redshifted. On the other half, the emission appears blueshifted with velocities ranging between 6 and $8 \mathrm{~km} / \mathrm{s}$. The axis of rotation would have an inclination of about $\sim 50^{\circ}$ with respect to the north (see Figure 1 Left). From the total velocity difference we can deduce that the possible observed rotation velocity is of the order of $\sim$ $6 \mathrm{~km} / \mathrm{s}$ 
For IRC+10011, see Figure 2, the same behavior is observed. The systemic velocity of the source is $\sim 8 \mathrm{~km} / \mathrm{s}$. We can see that one half of the spots, located at the North-East, appears generally redder than the maser spots appearing in the South-West. Interpreting this velocity in the case of the rotation of the inner shell, we would find a rotation velocity of $\sim 8 \mathrm{~km} / \mathrm{s}$ with an inclination of the rotation axis of about $45^{\circ}$ (with respect to the North).

\section{References}

Bujarrabal V. \& Nguyen-Q-Rieu 1981, A\&A, 102, 65

Bujarrabal V., Alcolea J.,\& Planesas P. 1992, A\&A, 257, 701

Bujarrabal V., Alcolea J., \& Neri R. 1998, ApJ, 504, 915

Desmurs J.F., Bujarrabal V., Colomer F.,\& Alcolea J. 2000, A\&A 360, 189 "VLBA observations of $\mathrm{SiO}$ masers: arguments in favor of radiative pumping mechanisms"

Diamond P.J., Kemball A.J., Junor W., Zenzus A., Benson J.,\& Dhawan V. 1994, ApJ, 430, L61.

Greenhill L.J., Colomer F., Moran J.M., Backer D.C., Danchi W.C., \& Bester M.A. 1995, ApJ, 449, 365.

Miyoshi M., Matsumoto K., Kameno S., Takaba H., \& Iwata T. 1994, Nat. 371, 395

Siess, L. \& Livio M. 1999, MNRAS, 304, 925.

Soker, N. 1998a, ApJ, 496, 833.

Soker, N. 1998b, MNRAS, 299, 1242.

Trammell S.R., Dinerstein H.L., \& Goodrich R.W. 1994, AJ, 108, 984

Van Winckel H., Waelkens C., \& Waters L.B.F.M. 1995, A\&A, 193, L25 